\title{
THE DEVELOPMENT OF INTEGRATED OBJECT-BASED ANALYSIS OF EO DATA WITHIN UK NATIONAL LAND COVER PRODUCTS.
}

\author{
G.M. Smith \\ Section for Earth Observation, Centre for Ecology and Hydrology, Monks Wood, Abbots Ripton, Huntingdon. PE28 2LS United \\ Kingdom.gesm@ceh.ac.uk
}

KEY WORDS: Land cover, National, Object-based, Generalised cartography

\section{ABSTRACT:}

The United Kingdom (UK) undertakes a survey of its countryside which have been accompanied by national land cover maps derived from Earth Observation data which have exploited leading edge analysis methods of the day. The Land Cover Map of Great Britain, in 1990 was a relatively simple pixel-based classification while the Land cover Map 2000 adopted an object-based approach. The objects, land parcels were derived by automated segmentation of the input image data and had a minimum mappable unit of 0.5 ha. Both of the above land cover products have been extremely successful, with in excess of 300 users. There have of course been problems with these products and these are mainly associated with the data models which have were somewhat abstract from reality. Preparations are now underway for a further update of the UK national land cover product which will again be object-based, but this time it is hoped that digital cartography can be adapted to give an object structure which more accurately reflect the true structure of the landscape. A feasibility study has demonstrated the key processes required to achieve the generalisation. The use of such a spatial structure will increase the potential user community and possibilities for integration of the next UK land cover product.

\section{BACKGROUND}

The United Kingdom (UK) undertakes a assessment of its landscape at intervals of 8 to 10 years known as the Countryside Survey (CS) (Haines-Young et al., 2003). The main component of the CS is a field survey where approximately $5601 \mathrm{~km}$ squares are visited for detailed ground-based measurements. The last two CSs have been accompanied by national land cover maps derived from Earth Observation (EO) data. These maps have developed over time exploiting leading edge analysis methods while maintaining a focus on operational requirements of a national mapping exercise.

The first of these, the Land Cover Map of Great Britain (LCMGB) in 1990, was a relatively simple pixel-based classification using Landsat Thematic Mapper (TM) data (Fuller et al., 1994). Multi-temporal TM data was used to maximise the amount of land cover discrimination that could be achieved, as certain land covers change on a seasonal basis. The data were classified with a conventional per-pixel implementation of a maximum likelihood approach and low level knowledge-based corrections were applied using simple masks.

\section{OBJECT-BASED LAND COVER MAPPING}

The LCMGB was extremely successful, but the pixel-based approach gave an arbitrary grid structure for the landscape. The pixel-based approaches also incorporated noise and unwanted natural variation into the classification resulting in a speckled product with little if any information on landscape structure. This situation encouraged the development of object-based approaches which analysed the EO data in units representative of real world features.

The Classification of Environment with Vector and RasterMapping (CLEVER-Mapping) project in the late 1990s developed an object-based classification procedure (Figure 1). The object-based approach avoided the mixed pixels at the edge of each object which were often misclassified in conventional per-pixel approaches due to their mixed spectral signatures for adjacent cover types (Dean and Smith, 2003). The spectral response in each image band was averaged for the core pixels only within each object to minimise noise and unwanted natural variation. The averaged spectral responses were then applied to a standard maximum likelihood algorithm and the resulting classification attached to the object as a whole.

The object-based structure allowed different EO data types to be combined and a broad range of non-EO data to be included as attributes on the object. The later were used to perform complex knowledge-based enhanced. For instance, objects with elevations greater than a few metres could be excluded from the intertidal habitats and soil type could be used to refine the seminatural grassland types recorded. The topologically structured objects also allowed advanced spatial context enhancements to be applied. For example, it is likely that small patches of arable completely surrounded urban are correct and bare ground in a coniferous forest context is more likely to be felled forest.

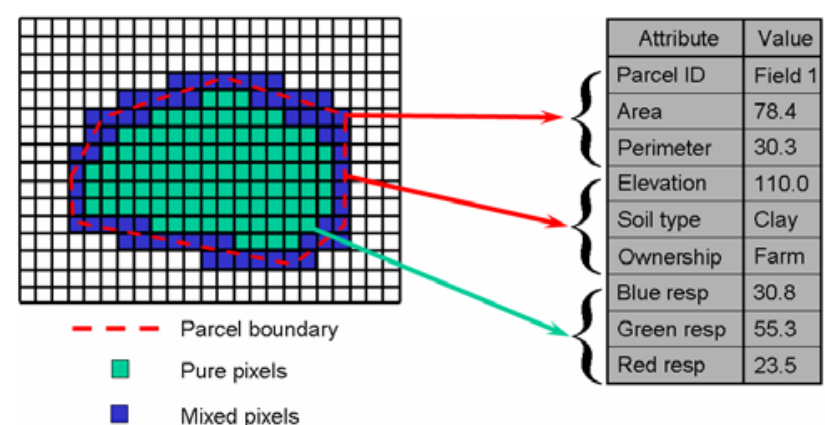

Figure 1. Schematic of the object-based classification approach developed during CLEVER-Mapping.

\subsection{Land cover map 2000}

An update of LCMGB was produced between 1998 and 2001, referred to as Land cover Map 2000 (LCM2000), which adopted an object-based approach (Fuller et al., 2002). A suitable object, land parcel, data set was not available nationally in the UK at the time of production so segmentation procedure was applied to the image data to generate a set of objects. The resulting spectral land parcels (Figure 2) related in the main to fields, woods, lakes etc. and had a minimum mappable unit (MMU) of 0.5 ha and a minimum feature width (MFW) of $25 \mathrm{~m}$. The final data set contained 6.6 million objects covering the $\sim 240000$ $\mathrm{km}^{2}$ of the UK. 


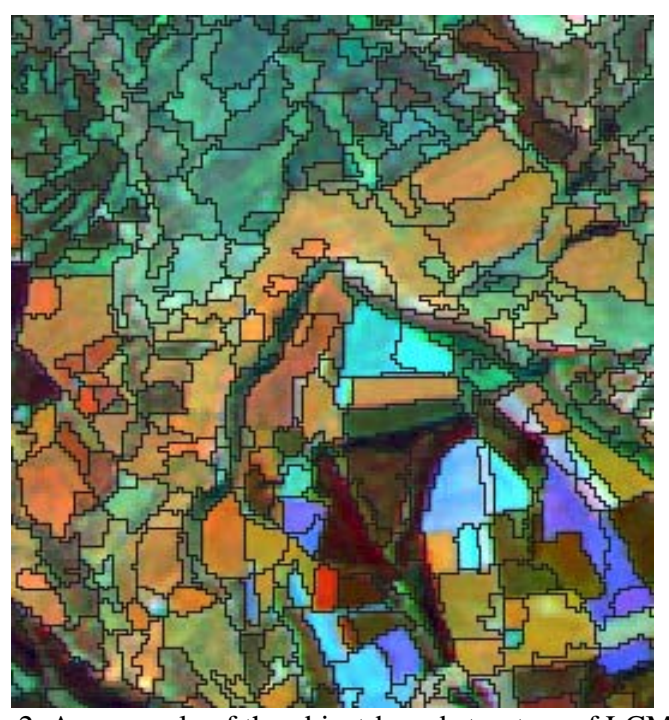

Figure 2. An example of the object-based structure of LCM2000 derived from image segmentation.

The land parcels were classified (Figure 3) using the procedures similar to those developed during CLEVER-Mapping to map the widespread examples of the UK Biodiversity Action Plan Broad Habitats. Each land parcel carried a rich set of parcellevel metadata as well as the resulting Broad Habitat as part of a hierarchical land cover class scheme.

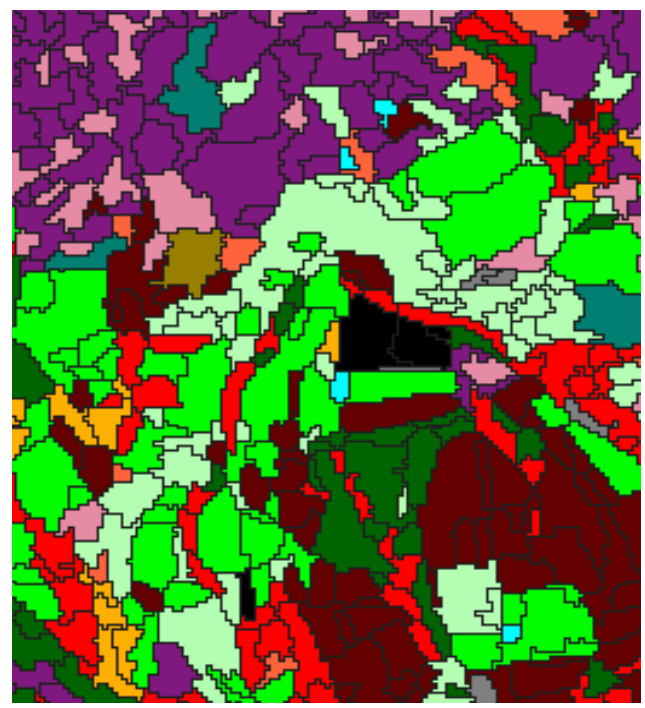

Figure 3. An example of the completed LCM2000 map labelled with Broad Habitats.

\subsection{Issues with previous products}

Both of the above land cover products have been extremely successful, with in excess of 300 user for each one. They have been used in a broad range of applications from syudies of national carbon budgets to the locating of telecommunications equipment.

There have of course been problems and criticisms with these products considering the broad user community they aimed to address. One of the main criticisms was associated with the data models which were somewhat abstract from reality.

The pixel model of LCMGB was an arbitrary grid unrelated to the actual landscape structure. The segmented land parcels of
LCM2000, although an improvement, reflect the spectral structure of the landscape rather than the presence of true boundary features. For instance, in LCM2000, two adjacent wheat fields could be combined into a single object by the segmentation process even though they may be owned by different farmers. Even if a boundary feature existed between them it would need to be spatially and spectrally significant at the spatial resolution of the image data to cause the segmentation algorithm to initiate a new object. Conversely, single fields many contain natural and acceptable variability which causes the segmentation algorithm to erroneous initiate a new object, giving multiple objects per field. For instance, a crop may progressively come into flower across a field and over time and the pattern of flowering could be captured by the image data and then recorded as spurious objects. The pixelated nature of the objects was also found to cause problems when comparing other data sets which represented diagonal boundaries in a more conventional manner.

The above considerations suggested that a new approach to the creation of the object, land parcel, framework should be developed which avoided the use of image data as far as possible.

\subsection{Development of real world object approach}

During the CLEVER-Mapping project a large scale prototype mapping exercise had been undertaken by producing a land cover map for the island of Jersey in 1997 (Smith and Fuller, 2001). The island government had digital cartography available for an area of approximately $215 \mathrm{~km}^{2}$, but this was too detailed to integrate with standard EO data sets (Figure 4). It was therefore necessary to generalise the digital cartography before the object-based classification could be applied. Unfortunately, at the time, the only means of doing this generalisation was by manually editing the line work and building objects from the disconnected lines. The process took around 2 person months and was therefore impractical for larger areas such as the UK.

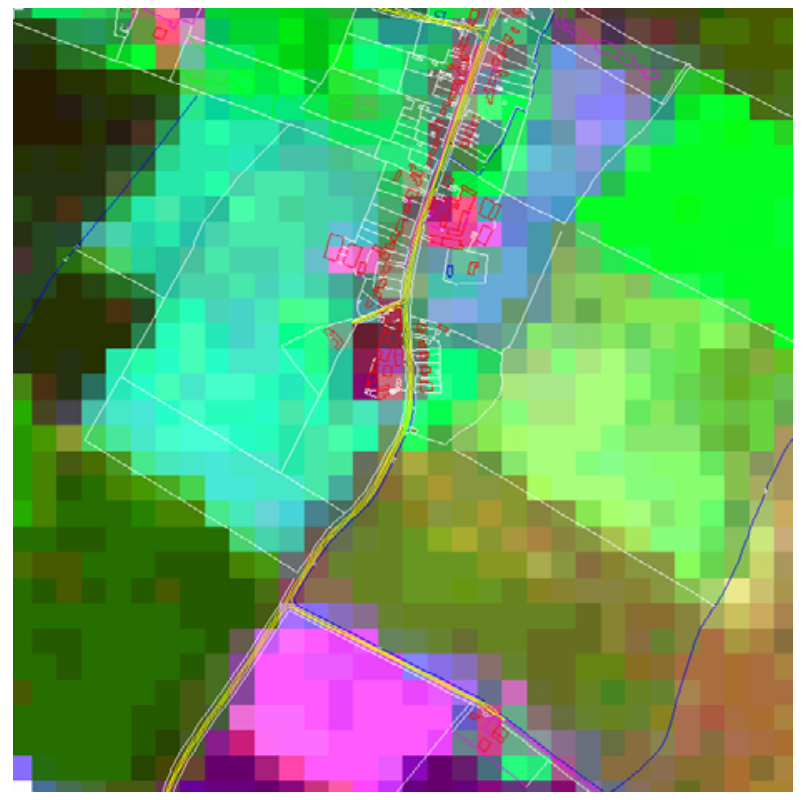

Figure 4. Detailed digital cartography overlaid on a $25 \mathrm{~m}$ spatial resolution satellite image. 
The resulting product (Figure 5) was of exceptional spatial quality compared to pixel-based and segment-based equivalents. The thematic accuracy was improved above that of the pixelbased approach and the relationship to existing cartography improved the usability and opportunities for integration with other data sets and within existing business systems.

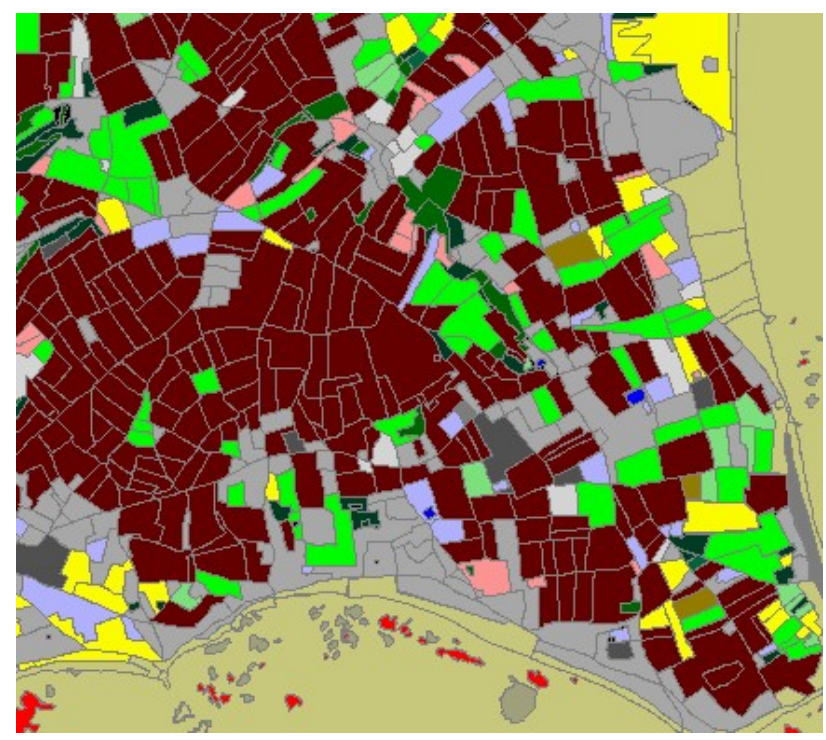

Figure 5. A section of the 1997 Land Cover Map of Jersey.

Preparations are now underway for a further update of the UK national land cover product with a target summer of 2007. This product will again be object-based, but this time it is hoped that digital cartography can be adapted to give an object structure which more accurately reflect the true structure of the landscape.

Since the release of LCM2000, the Ordnance Survey (OS) of Great Britain have produced a product called MasterMap (MM) by topologically structuring existing digital line work (similar to the data used in Jersey). The structuring of the data produces land parcels / real world objects rather than disconnected line work. This dataset is still far too detailed for effective integration with EO data with a $25 \mathrm{~m}$ spatial resolution, but is suited to automated generalization. It is therefore proposed to base the spatial structure of the next UK land cover product on a generalized version of MM.

\subsection{LCM2007 feasibility study}

To make this aim a reality it was necessary to develop an automated approach to the generalisation, so that large areas could be processed cost effectively and in a timely manner. A feasibility study was undertaken by Laser Scan of Cambridge, UK which developed and demonstrated the key processes required to achieve the generalisation. The specification for the generalised MM was based on the LCM2000 spatial specification of 0.5 ha MMU and 25 m MFW. The first step of the generalisation was to classify the MM objects by their geometric characteristics (Figure 6) so that:

- $\quad$ red objects are less than the MMU and simple;

- $\quad$ light blue objects are less than the MMU but complex (e.g. fail MFW rules);

- yellow and dark blue objects are larger than the MMU but complex; and

- $\quad$ green objects are larger than the MMU and simple.

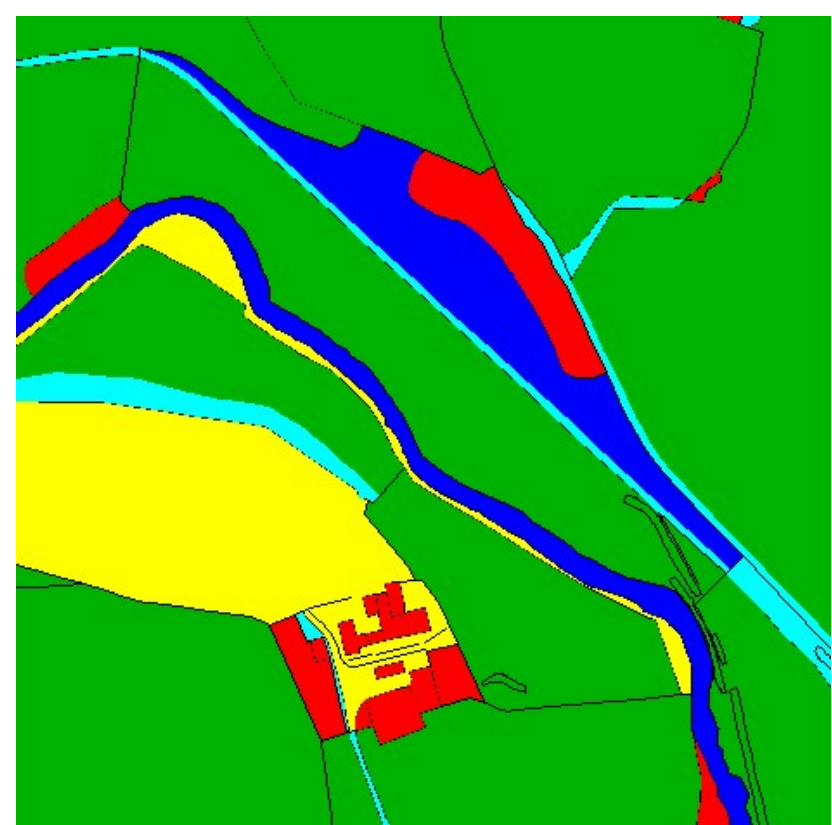

Figure 6. MasterMap object classified by their geometric characteristics.

Using this classification scheme the objects are merged and split iteratively until the data set only contains green objects which fit the specification. Figure 7 shows an example of the generalised $\mathrm{MM}$ data compared to an aerial photograph and Figure 8 shows it compared to a $25 \mathrm{~m}$ spatial resolution satellite image. Assessment by aerial photography interpreters has confirmed the quality and utility of the results. There are a few minor errors and ambiguities, but these will either be corrected in the next version of the procedure or highlighted for an operator to correct. In comparison with the satellite image data it can be seen that the generalised MM is fully aligned with the needs of an object-based analysis procedure. The area of woodland shows where the MM may lack some important boundaries, but these can be added for external data (forestry maps in this case) or by within object segmentation.

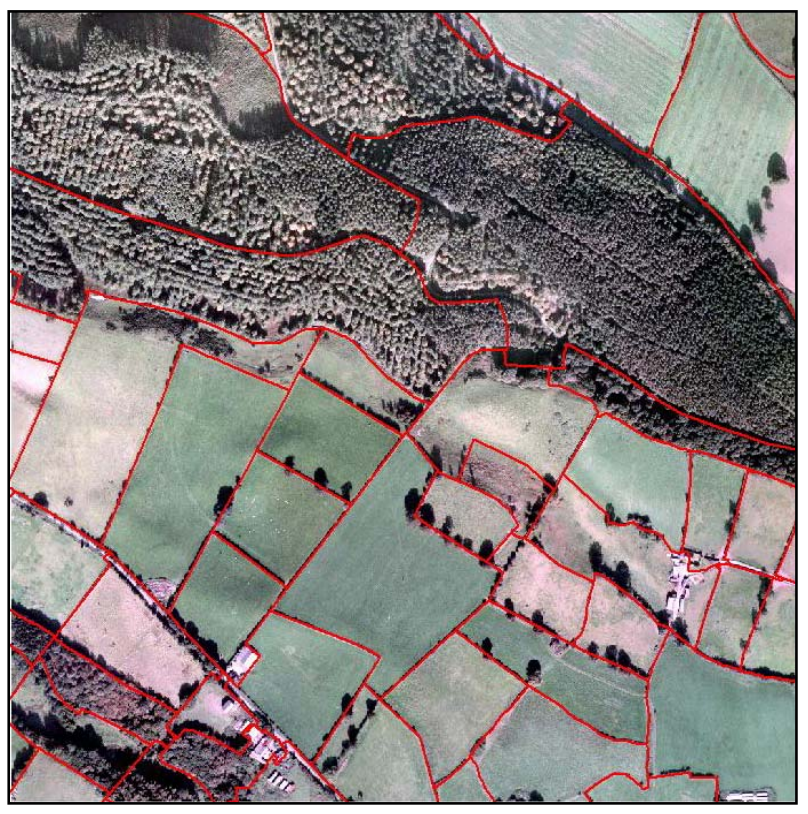

Figure 7. Generalised MasterMap data compared to aerial photography. 


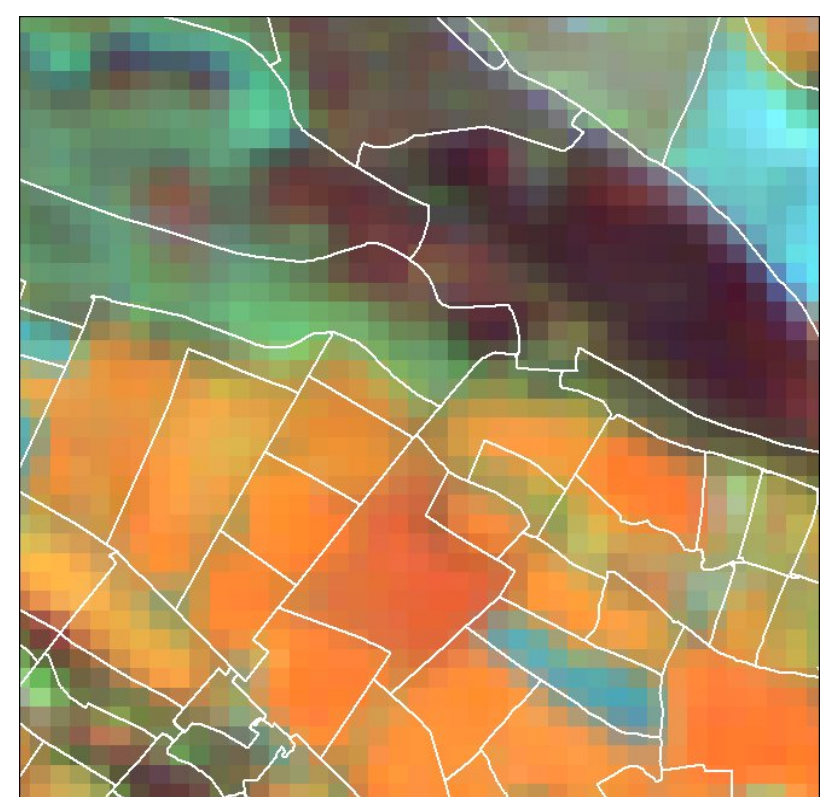

Figure 8. Generalised MasterMap data compared to a satellite image data.

The worked reported here was undertaken on $400 \mathrm{~km}^{2}$ area which included semi-natural, rural and urban landscapes. The generalisation appears to work well in all these landscapes, but will continue to be trailed at other sites across the UK prior to LCM2007 production. The approach has also been assessed in terms of its scalability and it has now been established that the approach can be applied to the whole of the UK in an automated fashion and reasonable timescale to provide nationally consistent generalised digital cartography to support land cover mapping with EO data.

The results obtained when classifying the generalised MM are very similar to those achieved in Jersey, but with little manual effort (Figure 9). When compared to the LCM2000 data for the same area the likely improvements in quality and usability are obvious (Figure 10).

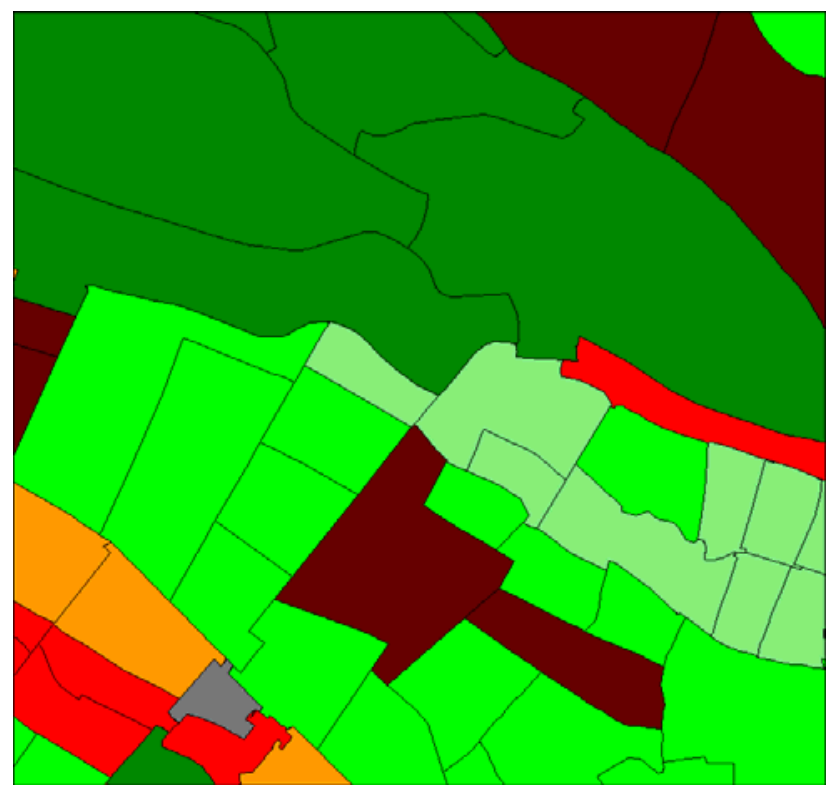

Figure 9. Generalised MM classified as Broad Habitats as in LCM2000.

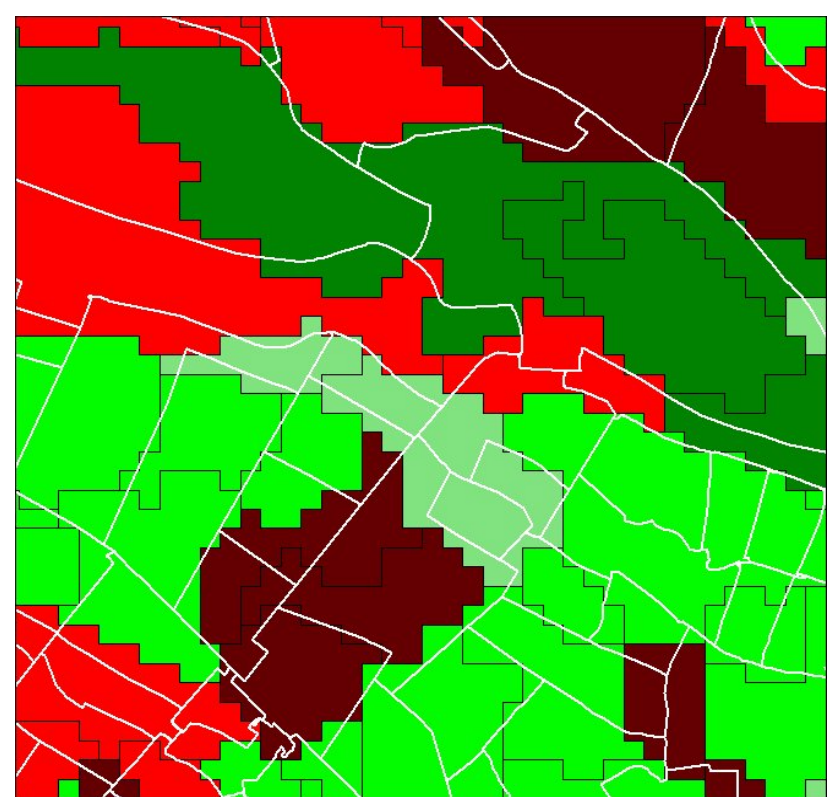

Figure 10. Generalised MasterMap compared to LCM2000 data of the same area.

\section{SUMMARY}

This paper described the background to the UK land cover products and the developments that have kept them at the leading edge of integrated object-based analysis of EO data. The work toward the production of LCM2007 will represent a major step forward for object-based land cover mapping. The use of such a spatial structure will increase the potential user community and possibilities for integration of the next UK land cover product.

\section{REFERENCES}

Dean, A.M. and Smith, G.M. 2003. An evaluation of per-parcel land cover mapping using fuzzy classification methods. International Journal of Remote Sensing, 24 (14), 2905-2920.

Fuller, R. M., Groom G.B. and Jones, A.R. 1994. The LandCover Map of Great-Britain - an Automated Classification of Landsat Thematic Mapper Data. Photogrammetric Engineering and Remote Sensing, 60(5): 553-562.

Fuller, R.M., Smith, G.M., Sanderson, J.M., Hill, R.A., and Thomson, A.G. 2002. The UK Land Cover Map 2000: Construction of a parcel-based vector map from satellite images. Cartographic Journal, 39(1): 15-25.

Haines-Young, R.H., Barr, C.J., Black, H.I.J., Briggs, D.J., Bunce, R.G.H., Clarke, R.T., Cooper, A., Dawson, F.H., Firbank, L.G., Fuller, R.M., Furse, M.T., Gillespie, M.K., Hill, R., Hornung, M., Howard, D.C., McCann, T., Morecroft, M.D., Petit, S., Sier, A.R.J., Smart, S.M., Smith, G.M., Stott, A.P., Stuart, R.C. and Watkins, J.W. 2000. Accounting for nature: assessing habitats in the UK countryside, DETR, London ISBN 1851124608

Smith, G.M. and Fuller R.M. 2001. An integrated approach to land cover classification: an example in the Island of Jersey. International Journal of Remote Sensing, 22(16): 3123-3142. 


\section{ACKNOWLEDGEMENTS}

The work described in this paper has been funded from a range of sources over time which can be identified through the sited papers. The most recent work on the feasibility of the generalising Ordnance Survey MasterMap was funded by the Natural Environment Research Council and undertaken by Laser Scan of Cambridge.
The author would also like to thank all those who have been involved in the UK national land cover mapping projects over the years for their inputs into the programme.

Further information about this programme can be found at http://www.ceh.ac.uk/sections/seo/lcm2000_home.html 